\title{
Design and Performance Analysis of Downlink in Space Communications System for Lunar Exploration
}

\author{
Wooju Lee $^{1}$, Kyongkuk Cho ${ }^{1}$, Dongweon Yoon ${ }^{1 \dagger}$, and Kwangmin Hyun ${ }^{2}$ \\ ${ }^{1}$ Dept. of Electronic and Communications Engineering, Hanyang University, Seoul 133-791, Korea \\ ${ }^{2}$ Dept. of Information and Telecommunication Eng., Gangneung-Wonju National University, \\ Gangwon-do 220-711, Korea \\ E-mail: dwyoon@hanyang.ac.kr \\ (Received October 6, 2009; Accepted January 14, 2010)
}

\begin{abstract}
This paper designs a data link between a Lunar Orbiter (LO) and an Earth Station (ES), and analyzes the downlink performance of a space communications system for lunar exploration, conforming to the recommendations by the Consultative Committee for Space Data Systems (CCSDS). The results provided in the paper can be useful references for the design of reliable communication link for the Korean lunar exploration in the near future.
\end{abstract}

Keywords: link budget, space communications, lunar exploration

\section{Introduction}

Korean government made clear that it would make efforts to carry out full-fledged research into space exploration with the aim of developing a Lunar Orbiter (LO) from 2017 to 2020 in the detailed implement guidance of the space development project established in 2007 (Lee 2009). To make the plan realized, basic researches into a space communication link are essential (Kim et al. 2009). However, local researches in Korea were focused on the near-earth satellite communication links and the researches on the deep space communications were hardly founded. This paper designs and analyzes the downlink between a LO and an Earth Station (ES) in space communications system for lunar exploration, and suggests requirements for the communication link design with conforming to international recommendations.

In general, among the losses in the calculation of a space communication link budget between the LO and the ES, the largest one is the free space loss comes from the distance between the earth and the moon. Furthermore, an accurate link model should be made up in order to analyze the performance in a more accurate way, with all the other elements influencing on signal quality. In this paper, we design the model of a space communications system considering almost all elements to affect the downlink performance of the space communications system between the LO and the ES, based on detailed requirements by CCSDS (the Consultative Committee for Space Data Systems, 2007), and verify the results with reference to the foreign operation cases of NASA (National Aeronautics and Space Administration) DSN (Deep Space Network) (Slobin 2006, Sniffin 2002, 2008). According

\footnotetext{
${ }^{\dagger}$ corresponding author
} 


\section{Lee et al.}

to the CCSDS, we assume that the communication links have the line of sight path between the LO and the ES for $\mathrm{S}, \mathrm{X}, \mathrm{Ku}$, and $\mathrm{Ka}$ bands, and an uncoded OQPSK signal is considered for a telemetry transmission. Also, a required target BER (Bit Error Rate) in the downlink space communications systems is assumed to be $10^{-5}$. We calculate the $E_{b} / N_{0}$ (Bit Energy to Noise Power Density Ratio) with the designed model for a downlink space communications system, and compare it with the required $E_{b} / N_{0}$ to satisfy the target BER. Then, we analyze the link performance between the LO and the ES depending on the data rates, the diameters of antennas, and the transmit powers in $\mathrm{S}, \mathrm{X}, \mathrm{Ku}$, and Ka bands.

The remainder of this paper is organized as follows: section 2 introduces a system model, section 3 analyzes the performance of the downlink space communications system, and the final section gives conclusions.

\section{System model}

In a receiver, the thermal noise is always existed caused by the motion of the internal electrons which are affected by the resistance and the temperature of the components. The thermal noise takes place in almost all components from the large-size antenna to the small-size components, and is represented as the equivalent noise temperature. In a communication system, the thermal noise is a basic reason for the performance degradation. Moreover, in a space communications system, the thermal noise contributes as a critically detrimental effect because the received signal strength is extremely low due to a long transmission distance. The thermal noise power $P_{N}$ can be expressed as

$$
P_{N}=k \cdot T_{S} \cdot B_{N}(W)
$$

where $k$ is the Boltzmann constant and equals $1.38 \times 10^{-23} \mathrm{~J} / \mathrm{K} ; T_{S}$ is the equivalent noise temperature, which presents various forms of noise caused in the circuit as the equivalent temperature; and $B_{N}$ is the equivalent noise bandwidth. The transmitted signals are weakened with various reasons until they reach the receiver due to following factors. Total received signal power should be calculated with statistically modeled loss elements.

\subsection{Free-Space Loss}

The free-space loss of a communication link in the line of sight is (Roddy 2006)

$$
[F S L]=32.44+20 \log _{10}(d)+20 \log _{10}(f)(d B)
$$

where $[X]=10 \log _{10} X(\mathrm{~dB})$; the unit of the distance $d$ between transmit and receive antennas is $\mathrm{km}$; and the unit of transmit frequency $f$ is $\mathrm{MHz}$.

\subsection{Atmospheric Attenuation}

When electromagnetic signals pass through the atmosphere of the earth, the signal power is weakened by energy absorption, scattering, and reflection of components in the atmosphere.

\subsection{Ionospheric Loss}

The atmosphere has many electrons and cations at an altitude of over $50 \mathrm{~km}$. This area is neutral on the whole, but generates a kind of ionized plasma, which is called ionosphere. In the ionosphere, free electrons which are non-uniformly distributed, make signal attenuation and change their phase resulting in one of the losses. 


\subsection{Rain Attenuation}

Rain attenuation drastically changes depending on space and time that it cannot be predicted easily. However, it is statistically estimated with the help of probability characteristics considering geological location, climate, topography, season and rainfall.

In low frequency bands, rain attenuation has little influences, but in high frequency bands, power loss rapidly increases. Especially, in a over $20 \mathrm{GHz}$ band, signal absorption and scattering by rainfall are so serious that severe attenuation occurs (ITU-R 2003). In case of designing a space communications system in high frequency band, it is required to carefully take the effect of rain attenuation into consideration due to geographical characteristics like frequent localized torrential downpours.

\subsection{Lunar Flux Density Loss}

Electromagnetic waves from the sun are reflected by the surface of the moon and go towards the earth. Among them, visible rays are seen with the naked eye. The rays of the sun are kinds of wide band electromagnetic waves. They act as an interference and effect on the communication link between the earth and the moon. The loss caused by them is assumed to be $4 \sim 6 \mathrm{~dB}$ (Johannsen et al. 1974, Park et al. 2009).

\subsection{Antenna Circuit Loss}

Antenna circuit loss is caused by imperfect impedance matching between components in antennas, cables, waveguides, filters, and couplers. The amount of circuit loss can be changed, depending on a way of designing and connecting hardwares. In this paper, we assume the circuit loss of the ES follows the standard of the NASA DSN. The circuit loss of the LO is assumed to be the same as that of the ES (Slobin 2008a,b, 2009).

\subsection{Pointing Loss}

In general, a space communications system uses a parabolic antenna for more antenna gains. Pointing loss statistically comes about $0 \sim 1 \mathrm{~dB}$, because the transmitter and the receiver antennas do not be correctly aligned each other.

\subsection{Calculation of $E_{b} / N_{0}$}

For an uncoded OQPSK signal, the required $E_{b} / N_{0}$ is $9.6 \mathrm{~dB}$ to meet the received BER of $10^{-5}$ in the AWGN (Additive White Gaussian Noise) channel (Proakis 2008). In order to obtain the link performance, we also consider the calculated $E_{b} / N_{0}$ with the designed model for a downlink space communications system. The received signal power $P_{R}$, is calculated as follows (Roddy 2006):

$$
\left[P_{r}\right]=\left[G_{T}\right]+\left[P_{T}\right]+\left[G_{R}\right]-\left[L_{T}\right](d B W)
$$

where $G_{T}$ is the gain of the transmitter antenna; $P_{T}$ is the transmit power; $G_{R}$ is the gain of the receiver antenna; and $L_{T}$ is the total loss between the transmitter and the receiver. $C / N$ (Carrier Power to Noise Power Ratio) can be obtained from the received power (3) and the thermal noise power (1) (Roddy 2006)

$$
[C / N]=\left[P_{r}\right]-\left[P_{N}\right](d B)
$$

In order to compare the calulated $E_{b} / N_{0}$ with the required one, $C / N$ in (4) should be converted into $E_{b} / N_{0}$. The relationship between $C / N, C / N_{0}$ (Carrier Power to Noise Power Density Ratio) and $E_{b} / N_{0}$ are (Roddy 2006)

$$
\begin{aligned}
& {\left[C / N_{0}\right]=[C / N]+\left[B_{N}\right](d B H z)} \\
& {\left[E_{b} / N_{0}\right]=\left[C / N_{0}\right]-\left[R_{b}\right](d B)}
\end{aligned}
$$

where $R_{b}$ is a bit rate. 
Table 1. High-speed downlink model.

\begin{tabular}{|c|c|c|c|c|c|}
\hline Classification & Unit & $\mathrm{S}$ band & $\mathrm{X}$ band & $\mathrm{Ku}$ band & Ka band \\
\hline System bandwidth & $\mathrm{MHz}$ & 26 & 26 & 26 & 26 \\
\hline Distance & $\mathrm{km}$ & 384,403 & 384,403 & 384,403 & 384,403 \\
\hline Transmit frequency & $\mathrm{MHz}$ & 2,295 & 8,420 & 12,200 & 32,000 \\
\hline \multicolumn{6}{|c|}{ Lunar orbiter } \\
\hline \multirow[t]{2}{*}{ Transmit power } & W & 20.0 & 20.0 & 20.0 & 20.0 \\
\hline & dBW & 13.0 & 13.0 & 13.0 & 13.0 \\
\hline Antenna diameter & M & 1.2 & 1.2 & 1.2 & 1.2 \\
\hline Antenna efficiency & & 0.7 & 0.7 & 0.7 & 0.7 \\
\hline Antenna gain & $\mathrm{dBi}$ & 27.7 & 38.9 & 42.2 & 50.5 \\
\hline Antenna circuit loss & $\mathrm{dB}$ & 0.6 & 0.4 & 0.3 & 0.25 \\
\hline Antenna pointing loss & $\mathrm{dB}$ & $3.2 \times 10^{-6}$ & $4.4 \times 10^{-5}$ & $9.3 \times 10^{-5}$ & $6.4 \times 10^{-4}$ \\
\hline \multicolumn{6}{|c|}{ Channel } \\
\hline Free space loss & $\mathrm{dB}$ & 211 & 222 & 225.9 & 234.2 \\
\hline Atmospheric attenuation & $\mathrm{dB}$ & 0.033 & 0.039 & 0.1 & 0.154 \\
\hline Ionospheric loss & $\mathrm{dB}$ & 0.2 & 0.2 & 0.2 & 0.2 \\
\hline Rain attenuation & $\mathrm{dB}$ & 0.0 & 1.0 & 4.7 & 19.2 \\
\hline Lunar flux density loss & $\mathrm{dB}$ & 5.34 & 5.4 & 5.0 & 3.96 \\
\hline \multicolumn{6}{|c|}{ Earth station } \\
\hline Antenna diameter & $\mathrm{M}$ & 34.0 & 34.0 & 34.0 & 34.0 \\
\hline Antenna efficiency & & 0.7 & 0.7 & 0.7 & 0.7 \\
\hline Antenna gain & $\mathrm{dBi}$ & 56.7 & 68.0 & 71.2 & 79.6 \\
\hline Antenna circuit loss & $\mathrm{dB}$ & 0.6 & 0.4 & 0.3 & 0.25 \\
\hline Antenna pointing loss & $\mathrm{dB}$ & 0.003 & 0.044 & 0.150 & 0.639 \\
\hline Noise temperature & $\mathrm{K}$ & 34.0 & 31.9 & 38.0 & 44.9 \\
\hline
\end{tabular}

\section{Design and link performance analysis of downlink in space communications system for lunar exploration}

High data rate is required to transmit the multimedia data collected by the LO through a downlink. Chang'e 1 of China and Selene of Japan for lunar explorations are planning to set the maximum downlink data rate as $2 \mathrm{Mbps}$ and $10 \mathrm{Mpbs}$, respectively (Hayashi et al. 2003, Hisahiro et al. 2005, Yu et al. 2005). Also, the NASA DSN sets the data rate up to $52 \mathrm{Mbps}$ (Tai 2007). Therefore, in this paper, we set the maximum downlink data rate as $52 \mathrm{Mbps}$ for the mutual compatibility of NASA DSN standards. The S, X, Ku, and Ka transmit frequency bands are also set as 2.295, 8.42, 1.22, and $32 \mathrm{GHz}$, respectively, conforming to the CCSDS standards.

\subsection{Design of downlink}

It is assumed that a Lunar Orbiter's Antenna (LOA) diameter is $1.2 \mathrm{~m}$; transmit power of the LO is $20 \mathrm{~W}$; an Earth Station's Antenna (ESA) diameter is $34 \mathrm{~m}$, referring to the BWG antenna DSS-24 in Goldstone, USA; and the noise temperature of the ESA in S, X, Ku, and Ka bands are distributed between 34 44.9 K (Slobin 2008a). The antenna gain of a parabolic antenna can be calculated as (Roddy 2006)

$$
[G]=10 \log _{10}\left\{\eta(10.472 f D)^{2}\right\} \quad(d B i)
$$

where $\eta$ means aperture efficiency, the value between $0.55 \sim 0.73 ; f$ is a frequency in $\mathrm{GHz}$; and $D$ is an antenna diameter in meter. Table 1 shows a model of the high-speed downlink in space communications system for lunar exploration, conforming to the NASA DSN and the CCSDS standards. Using from (3) to (6), $E_{b} / N_{0}$ can be obtained from the high-speed downlink model in Table 1. 


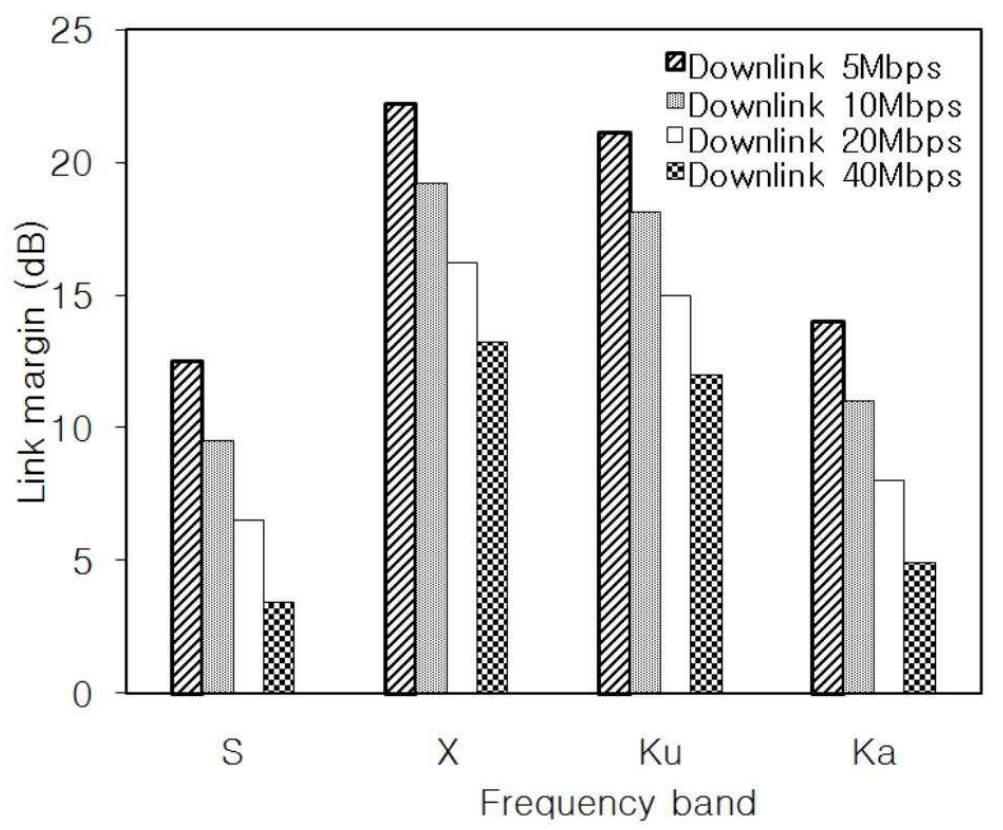

Figure 1. Link margins for the downlink data rates (under $52 \mathrm{Mbps}$ ).

\subsection{Link performances for data rates}

In this section, we analyze the link performances for data rates of the downlink with the referenced $34 \mathrm{~m} \mathrm{ESA}$ and $1.2 \mathrm{~m} \mathrm{LOA.} \mathrm{Figure} 1$ shows a link margins for the data rates from $5 \mathrm{Mbps}$ to 40 Mbps, based on the model in Table 1. Because we assumed uncoded OQPSK, frequency efficiency is $2 \mathrm{bps} / \mathrm{Hz}$. Thus the required bandwidths for the data rates of 5, 10, 20, and $40 \mathrm{Mbps}$ are 2.5, 5, 10 , and $20 \mathrm{MHz}$, respectively. From the Figure 1, we see that all data rates in the downlink have sufficient link margins for maintaining a reliable communication link in all frequency bands. As the data rate increases, however, the link margin decreases.

Figure 2 shows the link margins for the downlink data rates of $52 \mathrm{Mbps}, 100 \mathrm{Mbps}, 500 \mathrm{Mbps}$, and $1 \mathrm{Gbps}$ with system bandwidths of $26,50,250$, and $500 \mathrm{MHz}$, respectively in $\mathrm{S}, \mathrm{X}, \mathrm{Ku}$, and $\mathrm{Ka}$ bands. Only the data rate of $52 \mathrm{Mbps}$ can satisfy the link margin requirement in all frequency bands. In S band, data rates of $100 \mathrm{Mbps}, 500 \mathrm{Mbps}$, and $1 \mathrm{Gbps}$; and in $\mathrm{X}$ and Ku bands, data rate of 1 Gbps do not satisfy the condition for the reliable communication.

\subsection{Link Performance for antenna sizes}

Even we assumed that the reference diameter of the ESA is $34 \mathrm{~m}$, based on the DSS-24, we need to analyze the link performance for the ESA diameters of 26 and $70 \mathrm{~m}$, which are actually used for lunar exploration in the USA. Figure 3 shows the downlink performance for the ESA diameters of 26,34 , and $70 \mathrm{~m}$ at the data rate $52 \mathrm{Mbps}$ with the referenced diameter of $1.2 \mathrm{~m}$ in LOA. As the ESA diameter increases, the link performance becomes better due to the increased receiver antenna gain. The ESA diameters of 26, 34, and $70 \mathrm{~m}$ meet the sufficient link margins for the stable communication link in all bands.

Figure 4 shows the link performance for the LOA diameters of 0.6, 0.9, and $1.2 \mathrm{~m}$ at the data rate $52 \mathrm{Mbps}$ with the referenced diameter of $34 \mathrm{~m}$ in ESA. As the LOA diameter increases, costs of 
16 Lee et al.

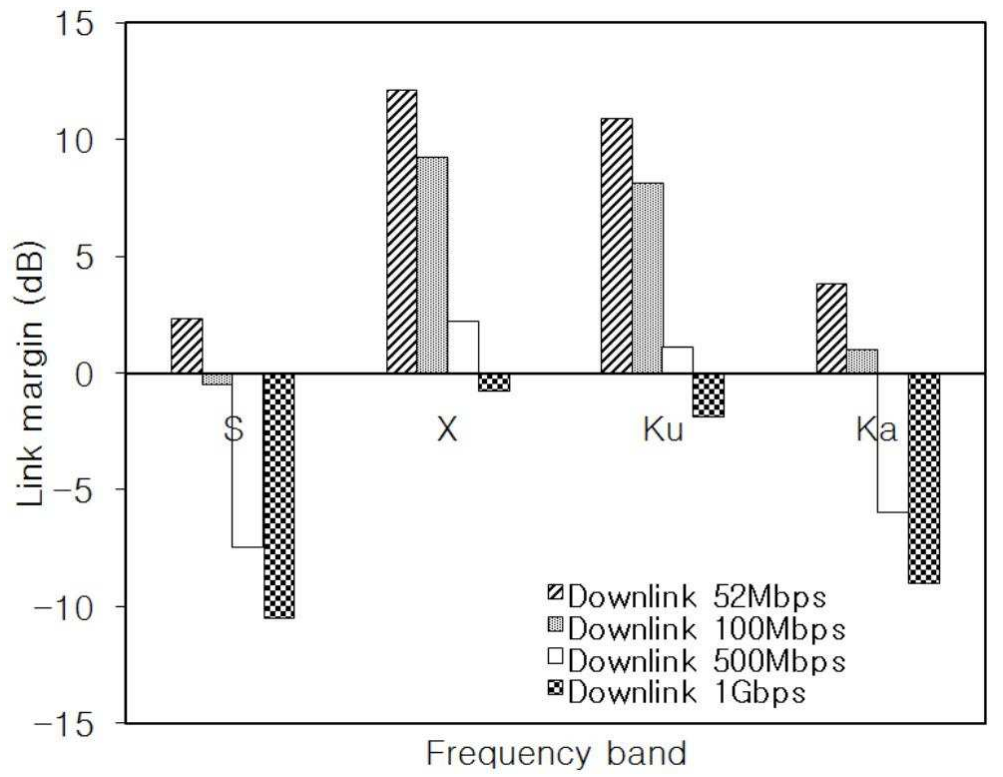

Figure 2. Link margins for the downlink data rates (over $52 \mathrm{Mbps}$ ).

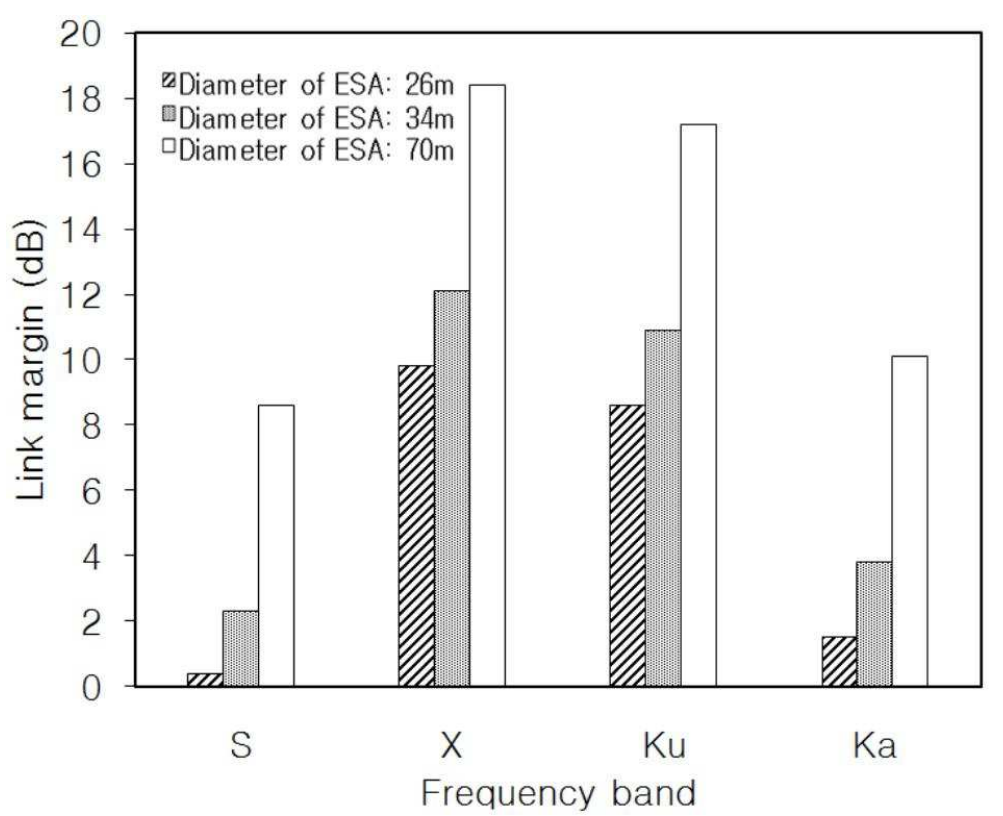

Figure 3. Link Performance for ESA diameters at the data rate $52 \mathrm{Mbps}$. 


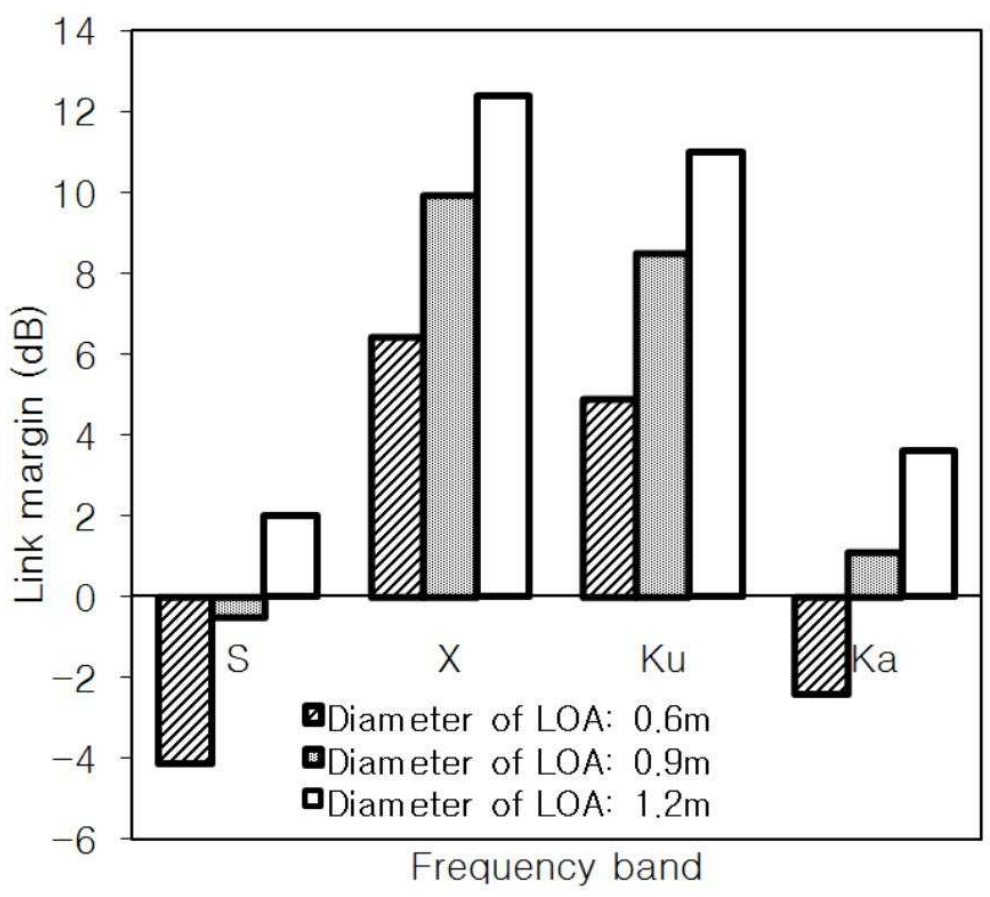

Figure 4. Link Performance for LOA diameters at the data rate $52 \mathrm{Mbps}$.

launching an orbiter will be increased. But the larger antenna size can provide better performance due to the increasing EIRP (Effective Isotropic Radiation Power) under a fixed transmit power. The LOA diameters of 0.6 and $0.9 \mathrm{~m}$ in $\mathrm{S}$ band; and $0.6 \mathrm{~m}$ in Ka are insufficient to establish the reliable communication link. The LOA diameters of $0.6,0.9$, and $1.2 \mathrm{~m}$ in X, Ku bands provide sufficient link margin.

\subsection{Link Performance for transmit powers of a LO}

Figure 5 shows the downlink performance when the transmit powers of a $\mathrm{LO}$ are 1, 10, 20, and $30 \mathrm{~W}$ at the data rate $52 \mathrm{Mbps}$ with the referenced diameters of $34 \mathrm{~m}$ in ESA and $1.2 \mathrm{~m}$ in LOA. In general, the LO's transmit power is a below $20 \mathrm{~W}$ because of the limited power capacity of the LO. But we also consider LO's transmit power of $30 \mathrm{~W}$ for comparison. As the transmit power of the LO increases resulting in higher EIRP, the link performance is improved. The LO's transmit powers of $1 \mathrm{~W}$ in all bands; and $10 \mathrm{~W}$ in $\mathrm{S}$ band do not meet the condition for reliable communication. The LO's transmit powers of 20,30 W provide the sufficient link margin for the stable communication link in all bands.

\subsection{Trade-offs between size of a LOA/an ESA and transmit power}

In sections 3.3 and 3.4, we analyzed downlink performances with different antenna sizes and transmit powers. In this section, we examine the trade-offs between antenna diameters and transmit powers at the data rate $52 \mathrm{Mbps}$, with the model in Table 1. Also, we focused on the trade-offs in the Ka band because the antenna gain is more sensitive than in any other band as shown in (7). Figure 6 shows the relationship between diameters of the ESA and the LOA for the different transmit powers $7,10,20$, and $30 \mathrm{~W}$ of the LO in the Ka band. Instead of the transmit power of $1 \mathrm{~W}$ of the LO in the 


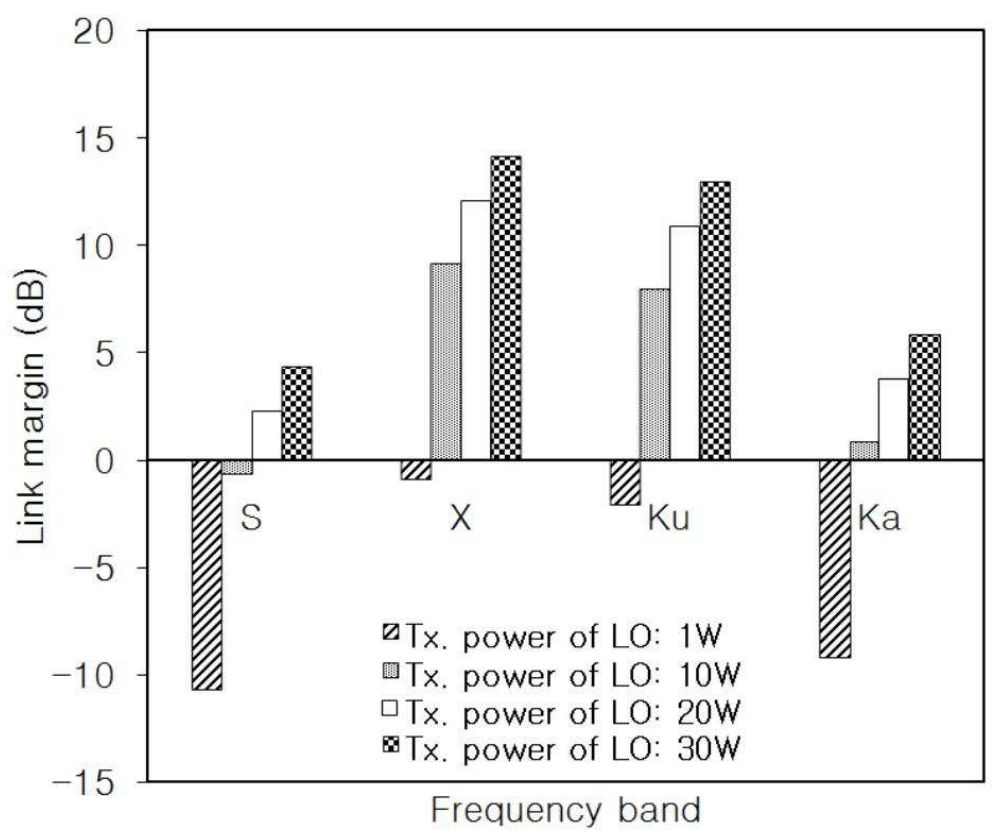

Figure 5. Link Performance for transmit powers of a $\mathrm{LO}$ at the data rate $52 \mathrm{Mbps}$.

previous section, we uses the transmit power of $7 \mathrm{~W}$ because the downlink performance is drastically degraded with the transmit power of $1 \mathrm{~W}$.

The increase in the transmit power of the LO is limited by the size of the solar arrays and available power. As the diameter of the LOA increases, the volume of the LO becomes larger resulting in higher launching costs. When the transmit power of the LO is $20 \mathrm{~W}$ and the diameter of LOA is $1.2 \mathrm{~m}$, the diameter of $11.4 \mathrm{~m}$ in an ESA is required for reliable communications. However, when the transmit power of the $\mathrm{LO}$ is $10 \mathrm{~W}$ and the diameter of LOA is $0.6 \mathrm{~m}$ with realistic limitations on the launched LO's volume, a large-size ESA diameter of at least more than $32 \mathrm{~m}$ is required.

If a channel coding technique is adopted, the antenna sizes and transmit powers of a $\mathrm{LO}$ and an ESA can be reduced as much as obtainable coding gain. Multiple antennas based on signal processing and adaptive modulation techniques are also expected to reduce the design burdens of a $\mathrm{LO}$ and an ES for reliable communications.

\section{Conclusions}

In this paper, we designed and analyzed the downlink between a LO and an ES in space communications system for lunar exploration, and suggested requirements for the communication link design with conforming to international recommendations. A simple way of improving the downlink performance is either to increase in the transmit power of a LO or in the diameters of an ESA or a LOA. However, there are some realistic limitations for the LO. The available power generated by solar cells of a LO are limited, so that the increase in transmit power of a LO has a limit. As the diameter of a LOA increases, the costs of launching a orbiter become higher. Considering these limitations, the trade-offs between the transmit power of a LO and the diameter of an ESA and a 


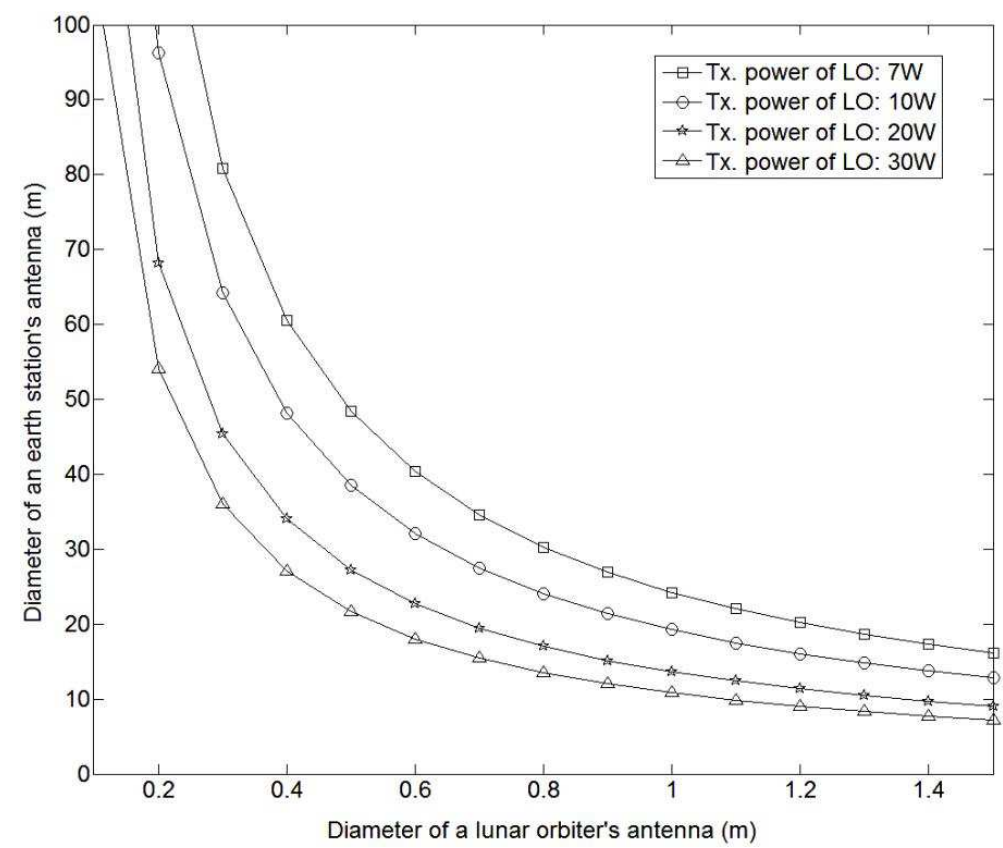

Figure 6. Diameters of an ESA and a LOA for transmit powers of a LO in the Ka band.

LOA were analyzed. When the transmit power of the LO is $20 \mathrm{~W}$ and the diameter of LOA is 1.2 $\mathrm{m}$, the diameter of $11.4 \mathrm{~m}$ in an ESA is required for reliable communications. However, when the transmit power of the LO is $10 \mathrm{~W}$ and the diameter of LOA is $0.6 \mathrm{~m}$ with realistic limitations on the launched LO's volume, a large-size ESA diameter of at least more than $32 \mathrm{~m}$ is required.

The results provided in the paper are expected to be primitive data for designing a space communications system between an ES and a LO for Korean lunar exploration in the near future.

Acknowledgments: This research was supported by NSL (National Space Lab) program through the Korea Science and Engineering Foundation funded by the Ministry of Education, Science and Technology (2009-0082437)

\section{References}

CCSDS 2007, 401.0-B

Hayashi, T., Nishimura, T., Takano, T., Betsudan, S. I., \& Koshizaka, S. 2003, ISAS, 82, 646

Hisahiro, K., Kato, M., Sasaki, S., Takizawa, Y., \& Mizutani, H. 2005, J. Earth Syst. Sci., 114, 771

ITU-R 2003, P.618-8

Johannsen, K. G. \& Koury, A. 1974, AES, 10, p.718

Kim, H.-D., Lee, J.-H., \& Sim, E.-S. 2009, in KSAS, ed. C.-D. Kong (Seoul: KSAS), p.713

Lee S.-R. 2009, KSAS Magazine, 3, 51

Park, D.-J., Kim, D.-J., \& Ahn, S.-I. 2009, IAC-09.A3.2INT.13

Proakis, J. G. 2008, Digital Communications (New York: McGraw-Hill), p.194

Roddy, D. 2006, Satellite Communications (New York: McGraw-Hill), p.352 
20 Lee et al.

Slobin, S. 2006, JPL, 810-005-105

Slobin, S. 2008a, JPL, 810-005-104

Slobin, S. 2008b, JPL, 810-005-103

Slobin, S. 2009, JPL, 810-005-101

Sniffin, R. W. 2002, JPL, 810-005-205

Sniffin, R. W. 2008, JPL, 810-005-201

Tai, W. S. 2007, JPL, 820-100

Yu, Z., Lu, L., Liu, Y., \& Dong, G. 2005, J. Earth Syst. Sci., 114, 795 